\title{
Productivity Improvement by Using Time and Motion Study Techniques in Lotus High Tech Company
}

\author{
H. H. Abdel-Maksoud*, A. El-Nikhaily**, R. I. Elsoeudy***, A. Z. Khafagy**** \\ *Demonstrator, Mechanical Department, Faculty of Industrial Education, Suez University, Suez, Egypt \\ **Professor, Mechanical Department, Faculty of Industrial Education, Suez University, Suez, Egypt \\ ***Professor, Production Engineering Department, Faculty of Engineering, Suez Canal University, Ismailia, Egypt \\ ****Professor, Production Engineering Department, Faculty of Engineering, Port Said University, Port Said, Egypt
}

Abstract

The purpose of this research is to improve productivity in Lotus High Tech Company (LHTC). This research used time and motion study techniques to improve the production process at LHTC. The research objectives towards accomplishing this study is to identify problems in the current method and improve it in terms of production time, number of process and production layout by proposing a new method to the manufacturing product. The current method in the manufacturing of garment in the production line involved some unnecessary steps, where the materials and components take a very long and roundabout path on their journey to the workstation, which adversely affect productivity. The proposed method eliminates these steps, which reduces production time, number of processes, and the overall increase in productivity is about $72.6 \%$.

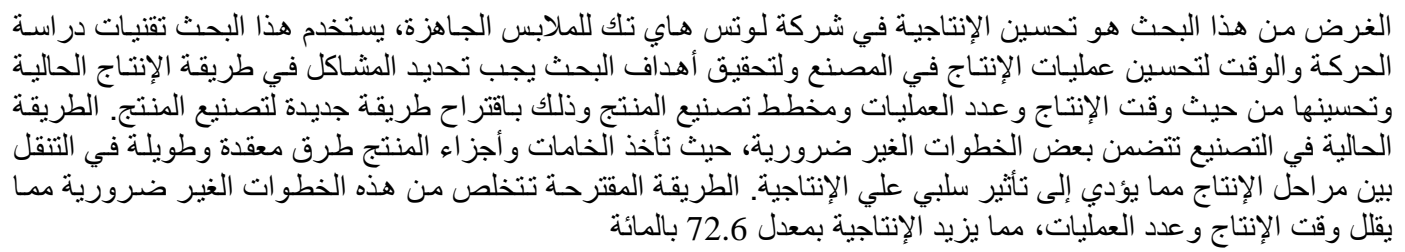

\section{INTRODUCTION}

Keywords: Time and Motion Study, Method Improvement, Production Processes, Flow Charts, Flow Process Analysis, Productivity.

Nowadays, the need to improve productivity is essential. Therefore, time and motion study is an important tool for controlling production and improving productivity in industrial organizations [1]. In studying any problem there should be a definite and ordered sequence. This sequence includes [1, 2, and 3]:

\section{Problem definition}

2. Obtain all the data relevant to the problem.

3. Examining the facts critically, taking into consideration the factors controlling the production method.

4. Develop new method to perform the task.

A conducted study by Gangopadhyay (2006) to organize production method by means of modifications in the existing method for enhancing productivity in sand core manufacturing. According to Gangopadhyay, it was found that the existing method of both types of sand core making involve some unnecessary steps, which may adversely affect productivity. The modified method eliminates these steps and the overall productivity in carbon dioxide sand core making and chemical sand core making increased by $8.5 \%$ and $30 \%$, respectively [4].

A conducted research was done by using time and motion study techniques to reduce the inspection time in the Motor Vehicle Periodic Inspection (MVPI), by Al-Saleh (2010). The main problem of this research was an inspection point (No. 1) which consumed more time in comparison with the other inspection points. Accordingly, this inspection point increases the flow time in the inspection lines. This research concluded that time and motion study techniques increase the productivity by $178 \%$ [5].

This research was performed to improve the production process and increase the productivity of the jeans Levi's Model 501 - line (No. 7) in Lotus High Tech Company (LHTC) station in Port Said, 
Egypt. In LHTC, the production lines are working in a classical routine mode without continuous evaluation of the production steps, which may adversely affect the productivity. Hence, they are looking to improve the productivity in production lines which operating in the brand Levi's Model 501. The time and motion study techniques are used directly as a means to improve productivity [6].

\section{Process Overview}

The product used in the present study is a garment Levi's Model 501. The major processes involved in manufacturing garment Levi's Model 501 consists of the following stages:

\subsection{Subassemblies}

Subassemblies are a collection of parts put together as a unit to be used in the making of a larger assembly or a final or higher item [7]. Subassemblies consist of the following components (Figure 1,2):

- Belt Loops: (4) release process, (5) making belt loops (6) belt loops fusing.

- Watch Pocket: (4) release process, (5) hem watch pocket (6) press watch pocket.

- Front Facing: (1L) release process, (2L) left front facing iron, (3L) attach left front facing on pocketing, (1L) examine left front facing for quality, (1R) release process, (2R) right front facing iron, (3R) mark watch pocket placement, (4R) attach right front facing on pocketing, (5R) attach watch pocket on right front facing, (1R) examine Right front facing for quality.

- Fly: (1L) release process, (2L) left fly buttonhole, (3L) serge left fly, (1R) release process.

- Front: (1L) release process, (2L) hem left front panel, (3L) attach left buttonhole fly piece, (4L) set front facing on left front, (5L) turn and topstitch front pockets, $(6 \mathrm{~L})$ close front pocket bag, (7L) turn front pocket bag, (8L) topstitch front pocket bag, (9L) stay notch at top and side, (10L) attach O.L. label at left front panel, (11L) serge left front panel, (1R) release process, $(2 \mathrm{R})$ set front facing on right front, (3R) turn and topstitch front pocket, (4R) close front pocket bag, (5R) turn front pocket bag, (6R) topstitch front pocket bag, (7R) stay notch at top and side, (8R) serge right front panel, (9R) attach right button fly with right panel, (4) top stitch fly \& hem crotch, (5) attach crotch, (6) join crotch, (2) examine the part for quality, (7) mark fly button \& front, (8) attach fly button

- Waistband: (4) release process, (5) mark CB waistband label

- Back Pocket: (1L) release process, (2L) left back pocket hemming, (3L) sewing arcuate at left back pocket, (4L) left Back pocket pressing, (1R) release process, (2R) right back pocket hemming, (3R) sewing arcuate at right back pocket, (4R) right Back pocket pressing.

- Riser: (1L) release process, (1R) release process

- Back: (1L) release process, (2L) attach left back pocket on back, (1L) examine the part for quality, (3L) attach back yoke to back (Left), (4L) serge left back panel, (1R) release process, (2R) attach right back pocket with global standard on back, (1R) examine the part for quality, (3R) attach back yoke to back (Right), (4R) serge right back panel, (4) seat seam attach, (2) examine the part for quality, (5) mark back, (6) bartack back BKT

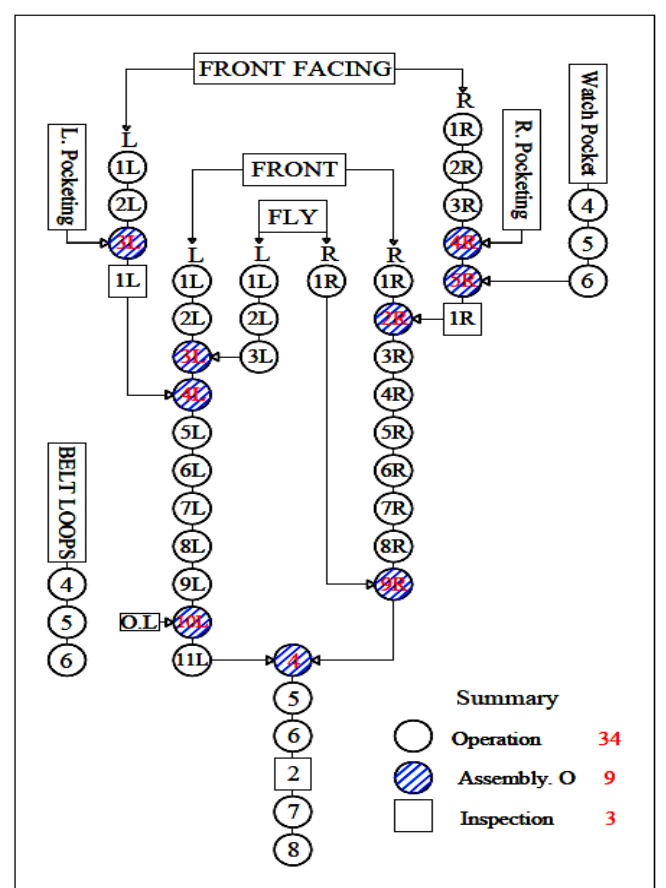

Figure 1 Operation Process Chart for the Front.

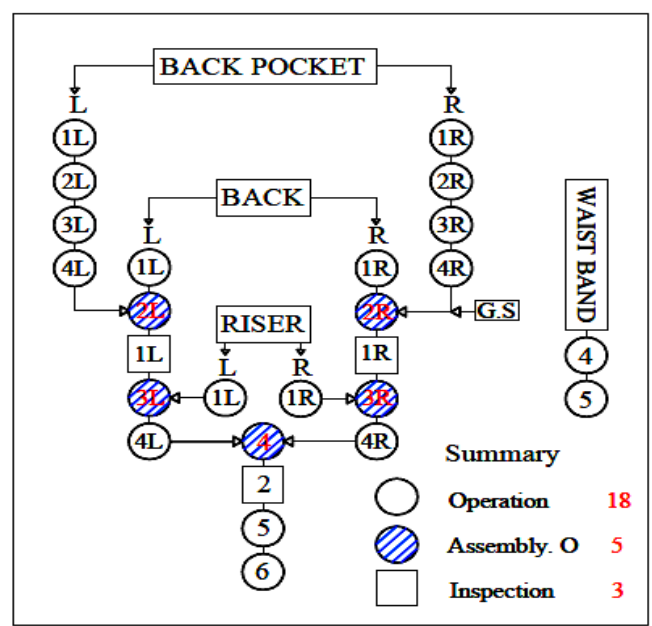

Figure 2 Operation Process Chart for the Back. 


\subsection{Final Assembly}

The final assembly shown in Figure 3 gives a broad overview for the final stages of assembling the final product (Levi's Model 501). The final assembly consists of the following stages:

(1) attach inseam, (2) top stitch inseam, (3) bartack front, (4) join side seam with Levi's label, (5) tack side seam, (6) cord stitch at side seam, (7) side seam busting, (8) bottom hemming, (9) turn pant, (10) attach CB waistband label. (1) Examine the part for quality, (11) finish waistband wnds (1), (12) finish waistband wnds (2), (13) attach rivets, (14) mark for back loops, (15) attach belt loops, (16) attach leather like label, (2) examine the part for quality. (17) Waist band buttonhole, (18) waistband button attach, (3) examine the product for quality, (19) closing fly buttons, (4) examine the product for quality (PO), (20) numbering of the products.

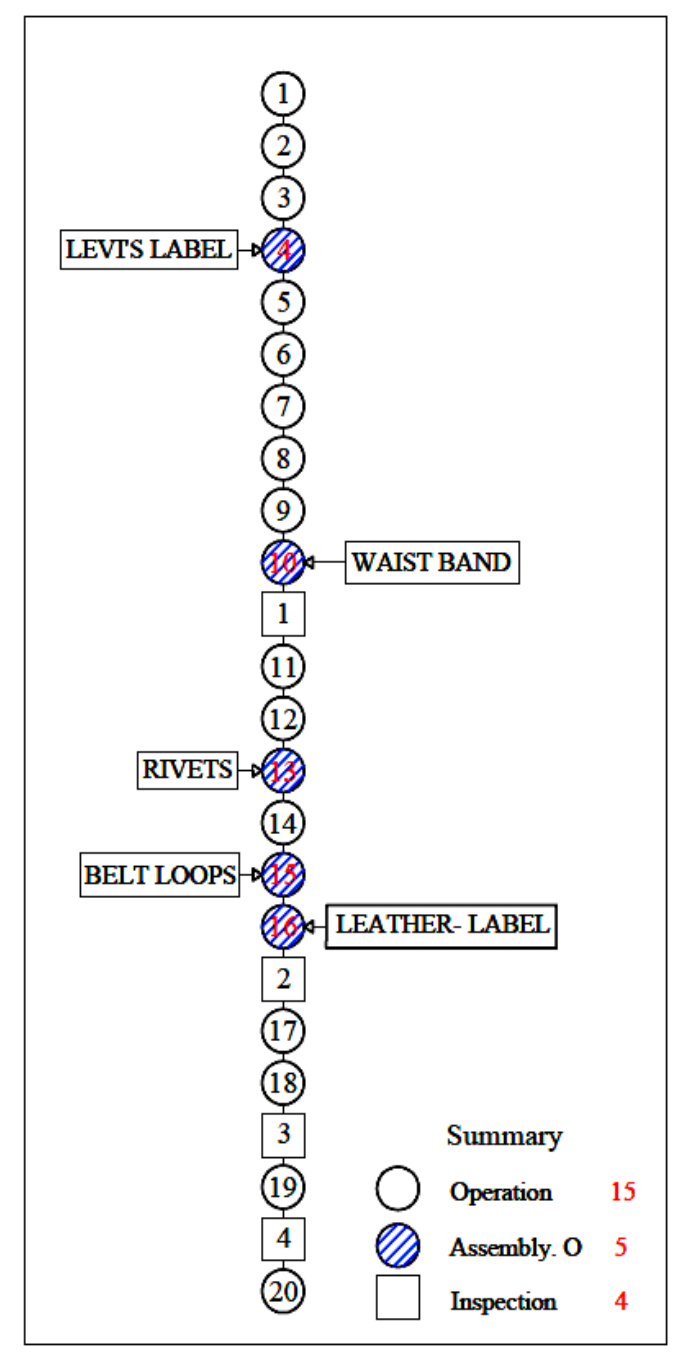

Figure 3 Operation Process Chart for the Assembly.

\section{Problem Statement \& Research Aim}

After continuously monitoring the production processes in line (No. 7) at LHTC, it was found that there are some problems in this plant such as:

1.The workspace is too small, and the production lines are too close to each other.

2. The passages to transport materials and products are too narrow and busy with handling equipment used in handling the parts. The movement of the forklift to transport the products of each production line is difficult which leads to low productivity.

3. The workers often use the method "Trial and Error", causing waste of time.

4. The materials and components take a very long and roundabout path on their journey to workstation.

Hence, it was found that there is a large percentage of the total production time as a delay and transportation of the materials and components.

The research aim is to improve the productivity in terms of production time, number of processes and production layout by proposing the new work process to LHTC. It is expected that the productivity, profit, and comfort of customers will increase while the production time, cost, and labor requirement will decrease $[8,9]$.

\section{Methodology}

In this research, time and motion study techniques were applied for tackling problems on all scales from the layout of complete factory to the smallest movements of workers in repetitive operations. These techniques can lead to reduction of redundant operations, and elimination of repeated steps, eventually resulting to reduced numbers of operations and production time [6, 8, and 9].

The following steps were employed for improving the productivity in the LHTC:

1. All activities have been observed, defined and recorded.

2. The necessary measurements for those activities have been taken by stopwatch (CASIO digital stopwatch HS-30W-1V).

3. The collected data in steps 1, 2 have been presented in different flow process charts and flow diagrams.

4. Examination of the actual situation and collected data critically.

5. Developing and defining new improved production method, which results in more productivity. 


\section{Results and Discussion}

The results obtained from the study were analyzed in the following to improve the productivity in terms of production time, number of processes and production layout by proposing the new better method to perform the task. These results are divided into two parts.

\subsection{Current Method}

Based on systematic observation of the current method, the collected data have been presented in flow process chart, and flow diagram. Analysis of flow process chart was conducted. All processes in current method shown in Figure 4 were examined.
From the flow diagram (Figure 4), the data was collected by measurement of time in each process.

This diagram was analyzed to show production method from the beginning (release process) to the end in production line (No. 7).

From the flow diagram (current method); it was found that the current method consists of 261 process, divided into 86 operation, 10 inspection process, while there are 128 transportation process, 32 delays, 5 storages, with overall processes time of about $630 \mathrm{~min}$, and the traveled distance about 2166 $\mathrm{m}$ for manufacturing 5 pieces from the product.

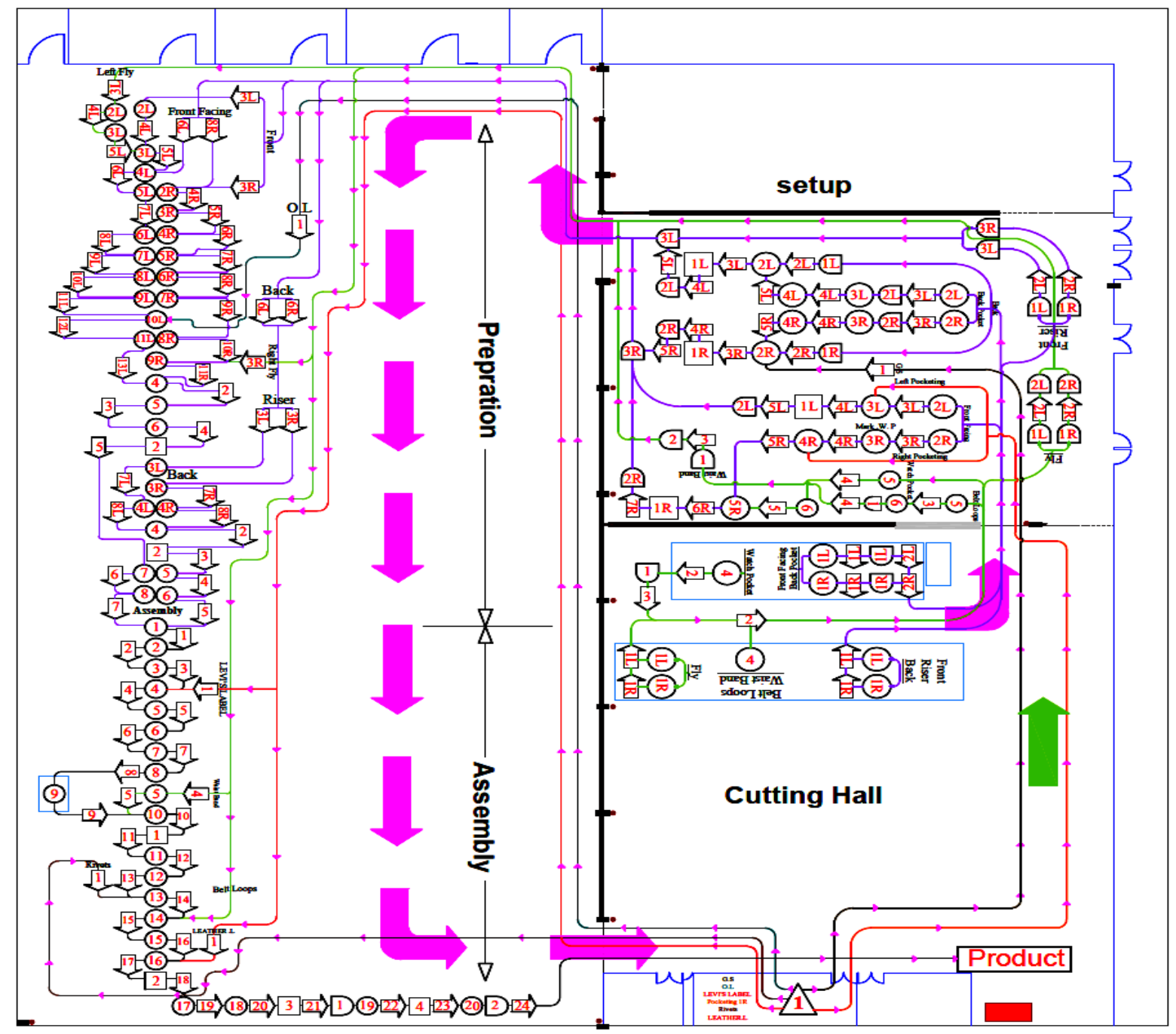

Figure 4 Flow Diagram of Levi's 501 in Lotus High Tech Company (current method)

\subsection{Proposed Method}




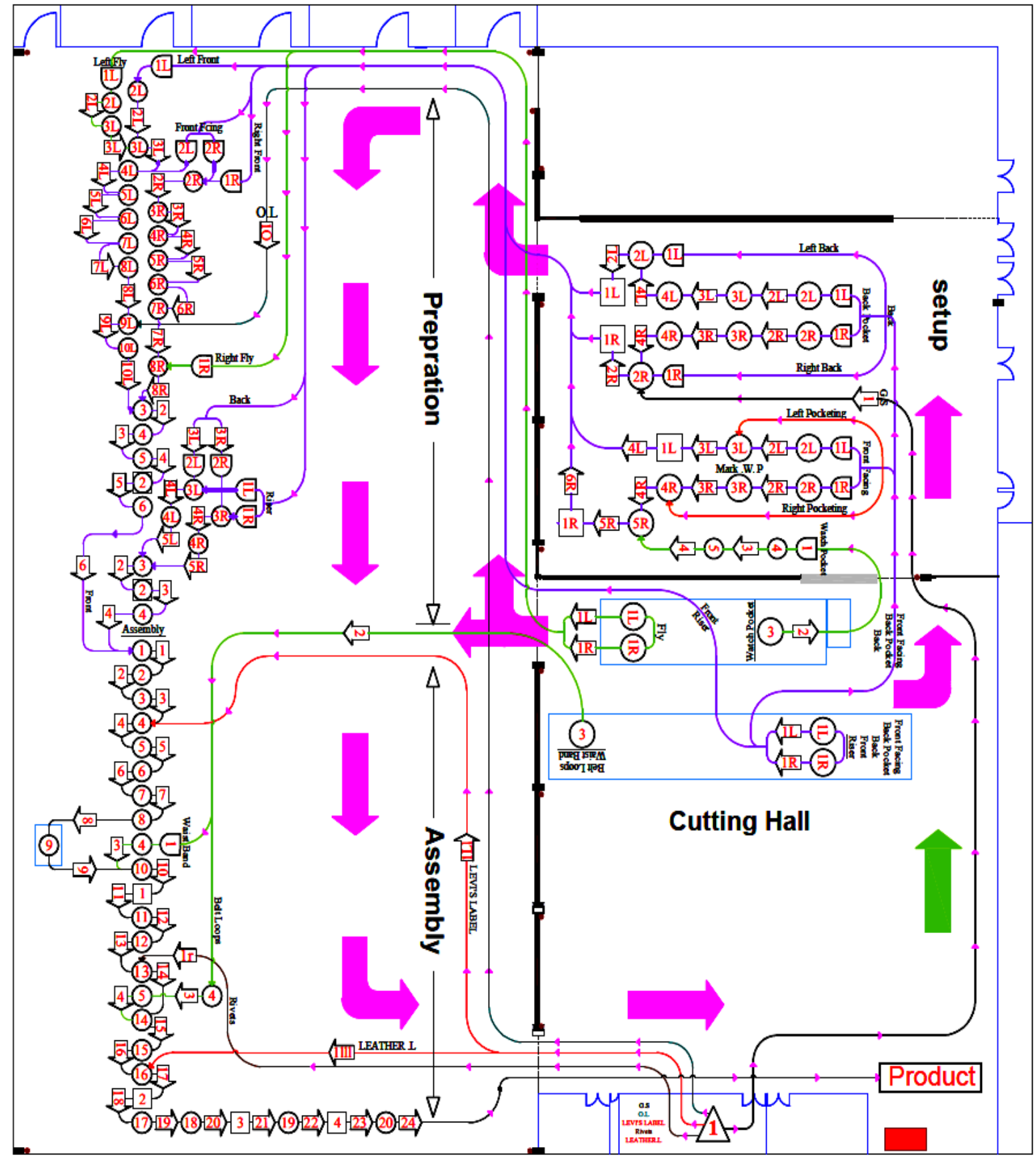

Figure 5 Flow Diagram of Levi's 501 in Lotus High Tech Company (proposed method)

From the observation, data collection and data analysis, it was develops production method to manufacture jeans (Levi's Model 501). In the proposed method, the unnecessary steps eliminated, combining some operations, and changes the arrangement of equipments in production department to reduce the total production time, shown in Figure 5. It was demonstrated that the number of processes can be decreased from 261 to 212 process. In the proposed method, it was found that the number of operations has been reduced from 86 to 82 . This has been done by combining some operations with other, which leads to reduce time of "operations" from 136 min to 128 min- making save of over 5 percent approximately. The transports have been reduced from 128 to 97 , by eliminating the unnecessary transportation, which leads to reduction of the transports time from $213 \mathrm{~min}$ to $112 \mathrm{~min}$ - making save of over 47 percent approximately. The inspections have been reduced from 10 to 8 by 
combining some inspections with operations (combined activity), which leads to a reduction time of "inspections" from 20 min to 15 min- making save of over 24 percent approximately. The number of "delays" has been reduced from 32 to 18 , by the eliminating the unnecessary delays, which leads a reduction in time of "delays" from $261 \mathrm{~min}$ to 105 min- making save of over 59 percent approximately. It is evident from the summary illustrated in Tables 1 and 2 that there have been considerable reductions in the number of "non- productive" activities which leads to reduce time of "processes" from $630 \mathrm{~min}$ to 364.6 min- making save of over 42 percent per 5 pieces from the product. Figure 6 shows the comparison between the current method and improved method in manufacturing of the product (Levi's Model 501).

Table 1: Change in number of processes after improvement.

\begin{tabular}{|c|c|c|c|c|}
\hline \multirow{2}{*}{ Process } & \multicolumn{3}{|c|}{ No. of processes } & \multirow{2}{*}{$\%$} \\
\hline & current & proposed & Saving & \\
\hline Storage $\quad \nabla$ & 5 & 5 & -- & 0 \\
\hline Operation $\bigcirc$ & 86 & 82 & 4 & 4.65 \\
\hline Transport $\Rightarrow$ & 128 & 97 & 31 & 24.2 \\
\hline Inspection $\square$ & 10 & 8 & 2 & 20 \\
\hline Delay $\quad \square$ & 32 & 18 & 14 & 43.75 \\
\hline Combined & -- & 2 & -2 & -100 \\
\hline Total & 261 & 212 & 49 & 18.77 \\
\hline
\end{tabular}

Table 2: Change in time of processes after improvement.

\begin{tabular}{|c|c|c|c|c|}
\hline \multirow{2}{*}{ Process } & \multicolumn{3}{|c|}{ Time (min) } & \multirow{2}{*}{$\%$} \\
\hline & current & proposed & Saving & \\
\hline Storage $\quad \nabla$ & -- & -- & -- & 0 \\
\hline Operation $\bigcirc$ & 136 & 128 & 8 & 5.9 \\
\hline Transport $\Rightarrow$ & 213 & 112 & 101 & 47.4 \\
\hline Inspection $\square$ & 20 & 15 & 5 & 25 \\
\hline D & 261 & 105 & 156 & 59.8 \\
\hline Combined & -- & 4.6 & -4.6 & -100 \\
\hline Total (min) & 630 & 364.6 & 265.4 & 42.13 \\
\hline
\end{tabular}

It is evident from the current method that jeans and its components follow an unnecessarily complicated path. Hence, there is discontinuity in flow of materials. After improvement the traveled distance has been reduced from about $2166 \mathrm{~m}$ to $1264 \mathrm{~m}$ making save of over 41 percent approximately as shown in Figure 7.

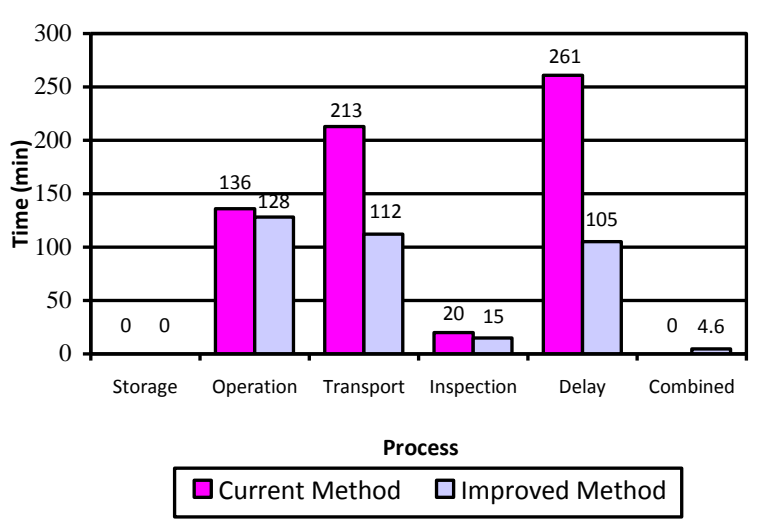

Figure 6 Comparison between the current method and proposed one.

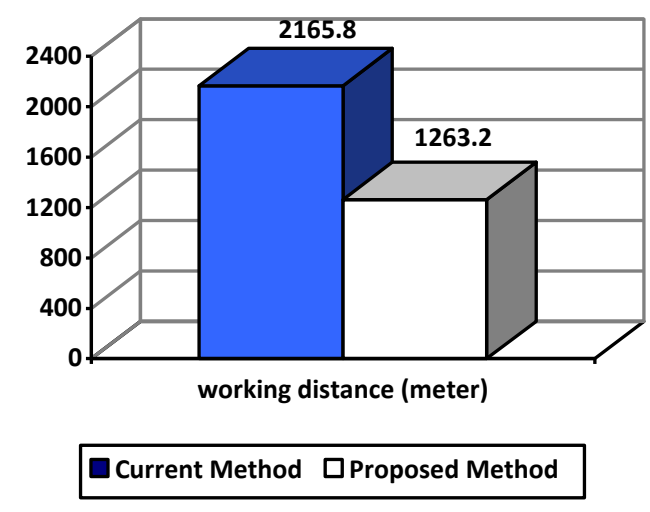

Figure 7 Reduction in traveled distance with proposed method.

In the improved method for manufacturing of the product (Levi's Model 501), the total time spent for manufacturing one piece (jeans) reduced from 126 $\min$ to $73 \mathrm{~min}$. As the workers made 1000 piece/day (current method), the total time saved in day was $53000 \mathrm{~min}$. Since the manufacture of one piece in the proposed method took $73 \mathrm{~min}$, additional 726 pieces could be prepared in the proposed method to be the output of the proposed method 1726 piece/day.

However, the increase in output in percent if Lotus High Tech Company applied the proposed method will be $72.6 \%$ by using the following formula $[2,5]$ : Increase in output \%

$$
\begin{gathered}
=\frac{\text { New method }- \text { Current method }}{\text { Current method }} \% \\
=\frac{1726-1000}{1000} \%=72.6 \%
\end{gathered}
$$




\section{Conclusion}

In this research, productivity is improved in Lotus High Tech Company (LHTC) by using time and motion study techniques. This research analyzed the current method using flow process charts and flow diagrams. The proposed method eliminates unnecessary steps, which leads to reduce production time, number of processes. The distances in the transportation of materials and components can be shortened from about $2166 \mathrm{~m}$ to $1264 \mathrm{~m}$ (making save of over $41 \%$ ). The total time spent for manufacturing one pieces (jeans) reduced from 126 $\min$ to $73 \mathrm{~min}$ (making save of over $42 \%$ ). The productivity in manufacturing garment Levi's Model 501 will increase by $72.6 \%$, if Lotus High Tech Company applied the proposed method.

\section{References}

[1] Barnes, R. M., "Motion and Time Study Design and Measurement of Work", New York: John Wiley \& Sons, $7^{\text {th }}$ ed, 1980.

[2] Kanawaty, G, "Introduction to Work Study", Geneva, International Labour Office, $4^{\text {th }}$ ed, 1992.

[3] Chang, B., "The Characteristics of Motion and Time Study in Taiwan's Electronics Industry and Their Relationship to Business Size", Doctor Thesis, University of Northern Iowa, 1994.

[4] Gangopadhyay, S. "Work Organization in Sand Core Manufacturing for Health and Productivity", International Journal of Industrial Ergonomics Vol. 36, 2006.

[5] Al Saleh, K. S., "Productivity Improvement of A Motor Vehicle Inspection Station Using Motion and Time Study Techniques", Journal of King Saud University, Engineering Sciences Vol 23, 2011.

[6] Bon, A. T., "An Impact Time Motion Study on Small Medium Enterprise Organization", Faculty of Technology Management, Universiti Tun Hussein Onn Malaysia, 2008

[7] Lee, H. H. "Cost/Benefit models for Investments in Quality Improvements", Doctor Thesis, The Pennsylvania State University, 2001.

[8] Sharma, S. C., "Plant Layout and Materials Handling", Khanna Publishers, $1^{\text {st }}$ ed, 2003.

[9] Islama, M., Hossain, M., and Islam, A., “A Comparative Method Study for Printing Time Reduction in Knitwear Manufacturing Company in Bangladesh: A Case Study", World Journal of Social Sciences, Vol. 2, 2012. 